\title{
Adaptive Particle Swarm Optimization via Velocity Feedback
}

\author{
Nobuhiro Iwasaki ${ }^{*}$ Student Member \\ Keiichiro Yasuda* Member
}

This paper describes an adaptive strategy for tuning the parameters of the PSO method based on an analysis of the dynamics of PSO. This adaptive tuning strategy is based on the results of an analysis of the dynamics of average velocity of the particles with successful search processes. The feasibility and advantages of the proposed adaptive PSO method are demonstrated through numerical simulations using a typical global optimization test problem.

Keywords: Global Optimization, Meta-heuristics, Particle Swarm Optimization, Adaptive Search

\section{Introduction}

Particle Swarm Optimization (PSO) method is one of the most powerful methods available for solving both unconstrained and constrained global optimization problems. The method was originally proposed by J. Kennedy et al. as an optimization method in 1995. Subsequent numerical experiments have demonstrated that the PSO method is efficient for a variety of global optimization problems with nonlinear and multi-peaked features ${ }^{(1)}$.

Meta-heuristics, which include the PSO method, are approximate algorithms designed to be applied to engineering problems. It is clearly desirable that these algorithms should be able to be applied to real optimization problems without the need for highly skilled labor.

However, to date their application has required significant time and labor for tuning the parameters, and so, from an engineering viewpoint, it is desirable to add the adaptability to these algorithms. The adaptability of an optimization method describes the method's ability to adaptively adjust its parameters.

\section{Particle Swarm Optimization}

2.1 Algorithm of PSO In the PSO algorithm, each particle has its own position, $x$, transfer vector (velocity), $v$, and own best position visited so far, pbest. Furthermore, all the particles share gbest, the best position visited by all the particles so far. A particle forms a new transfer vector (velocity) by linearly combining three vectors. In the $(k+1)$-th transfer, the $j$-th coordinate component of transfer vector of the $i$-th particle is manipulated according to the following equation:

$$
\begin{aligned}
v_{i j}^{k+1}= & w \cdot v_{i j}^{k}+c_{1} \cdot \operatorname{rand}_{1}() \cdot\left(\text { pbest }_{i j}^{k}-x_{i j}^{k}\right) \\
& +c_{2} \cdot \operatorname{rand}_{2}() \cdot\left(\text { gbest }_{j}^{k}-x_{i j}^{k}\right) \cdots \cdots
\end{aligned}
$$

where $w, c_{1}$ and $c_{2}$ are non-negative parameters which characterize the dynamics of particles; $\operatorname{rand}()$ is random number which is uniformly distributed in $[0,1]$; and $k$ determines the iteration number ${ }^{(1)}$.

\footnotetext{
* Tokyo Metropolitan University

1-1, Minamiosawa, Hachioji-shi, Tokyo 192-0397
}

Now each agent moves according to the following equation:

$$
x_{i j}^{k+1}=x_{i j}^{k}+v_{i j}^{k+1}
$$

2.2 Inertia Weights Approach Inertia Weights Approach (IWA) is one of the most famous strategies for tuning the parameters of PSO. In IWA, the value of the parameter $w$ is large at the beginning of search process, whereupon it gradually becomes smaller as the iteration advances. For example, the values $w=0.9$ (at the beginning of search process), $w=0.4$ (at the end of search process) and $c_{1}=c_{2}=2.0$ (constant), are recommended ${ }^{(1)}$.

Many numerical experiments have reported that IWA is effective for various kinds of optimization problems. By its strategy for the adjustment of parameter $w$, while a diversification in the search is realized in the initial stage, an intensification in search is also realized in the final stage of the search. However, from our numerical experiments, the search ability of IWA is reduced when the scale of the problem becomes large.

3. Information on Average Velocity of a Swarm

3.1 Index for Activity of Particles As it was described above, a particle transfers by using transfer vector $v_{i j}$ generated from Equation (1), therefore $\left|v_{i j}\right|$ is the transfer step-size. Since in this equation randomness is included, the transfer vector $v_{i j}$ fluctuates due to this randomness. The average of absolute value of velocity of all the particles can be used as an index to understand the activity of all the particles.

$$
v_{\text {ave }}=\frac{1}{m \cdot n} \sum_{i=1}^{m} \sum_{j=1}^{n}\left|v_{i j}\right| \ldots \ldots \ldots \ldots \ldots \ldots \ldots
$$

3.2 Relationship between the Success or Failure of Search and Average Velocity of Swarm If the value of parameters are not appropriate, the absolute value of velocity $\left|v_{i j}\right|$ can increase or decrease rapidly. Neither of these cases seems to be desirable for the search process. In order to confirm that, we tried some numerical simulations for some typical benchmark 


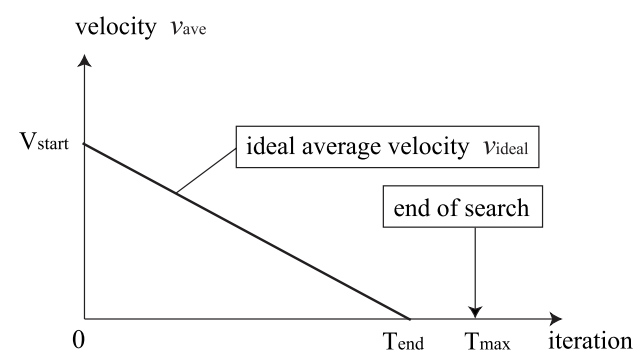

Fig. 1. Ideal average velocity of swarm for the proposed adaptive PSO.

problems. The conclusions we drew from the simulations are:

(a) In case $v_{\text {ave }}$ increases as the iteration advances, the search often ends in the failure,

(b) In case $v_{\text {ave }}$ decreases rapidly as the iteration advances, the search often ends in the failure,

(c) In case $v_{\text {ave }}$ decreases gradually as the iteration advances, a good solution tends to be obtained.

As indicated by these results, there is a qualitative relationship between the average velocity of the swarm and the success or failure of the search.

\section{Adaptive PSO using Velocity Information of Swarm}

Based on the fact that success or failure of a search is related to the average of absolute value of velocity, a new algorithm in which parameters are tuned to control the average velocity is proposed.

4.1 Algorithm of Adaptive PSO Although the search ability of IWA declines when it is applied to a large-scale problem, the strategy of IWA, that is the phase of searching gradually shifting from diversification to intensification with a progress of iteration, appears to be effective for optimization. Thus we propose a new algorithm in which parameters are tuned adaptively in order to maintain the ideal average velocity as shown in Fig. 1. In this algorithm, $c_{1}=c_{2}=1.3$ are constant.

If $v_{\text {ave }}$ is smaller than the ideal average velocity $w$ shifts to 0.9 ; this is a divergent value and $v_{\text {ave }}$ will increase. Otherwise, $w$ shifts to 0.8 ; this is convergent value and $v_{\text {ave }}$ will decrease.

$$
w^{k}= \begin{cases}0.9 & , v_{\text {ave }}^{k} \leq v_{\text {ideal }}^{k} \\ 0.8 & , v_{\text {ave }}^{k}>v_{\text {ideal }}^{k}\end{cases}
$$

The setting of these values is based on the stability analysis we have been carried out.

\subsection{Simulation results of Proposed Method}

$$
\begin{aligned}
& {\left[2^{n} \text { minima }\right]} \\
& \min f=\sum_{i=1}^{n}\left(x_{i}^{4}-16 x_{i}^{2}+5 x_{i}\right) \\
& \text { subj.to. } \quad-5 \leq x_{i} \leq 5 \ldots \ldots
\end{aligned}
$$

Table 1 shows the application results of both the proposed method and IWA to the benchmark $2^{n}$ minima problem as formulated Equation (5), where the number of population of the swarm is 20 . The values $w=0.9$ (at the beginning of the search process), $w=0.4$ (at the end of the search process) and $c_{1}=c_{2}=2.0$ (constant),
Table 1. Simulation results of the proposed method compared with IWA.

\begin{tabular}{c|c||c|c||c|c}
\hline \hline \multirow{2}{*}{$\begin{array}{c}\text { Dim. } \\
n\end{array}$} & \multirow{2}{*}{$\begin{array}{c}\text { Optimal } \\
\text { value }\end{array}$} & \multicolumn{2}{c||}{ Iteration=1000 } & \multicolumn{2}{c}{ Iteration=3000 } \\
\cline { 3 - 6 } & IWA & $\begin{array}{c}\text { Proposed } \\
\text { method }\end{array}$ & IWA & $\begin{array}{c}\text { Proposed } \\
\text { method }\end{array}$ \\
\hline 10 & -783 & -755 & -774 & -755 & -783 \\
20 & -1567 & -1394 & -1455 & -1456 & -1505 \\
30 & -2350 & -1713 & -2040 & -2083 & -2106 \\
40 & -3133 & -1327 & -2635 & -1923 & -2726 \\
50 & -3917 & -1457 & -2750 & -1350 & -3257 \\
\hline \hline
\end{tabular}

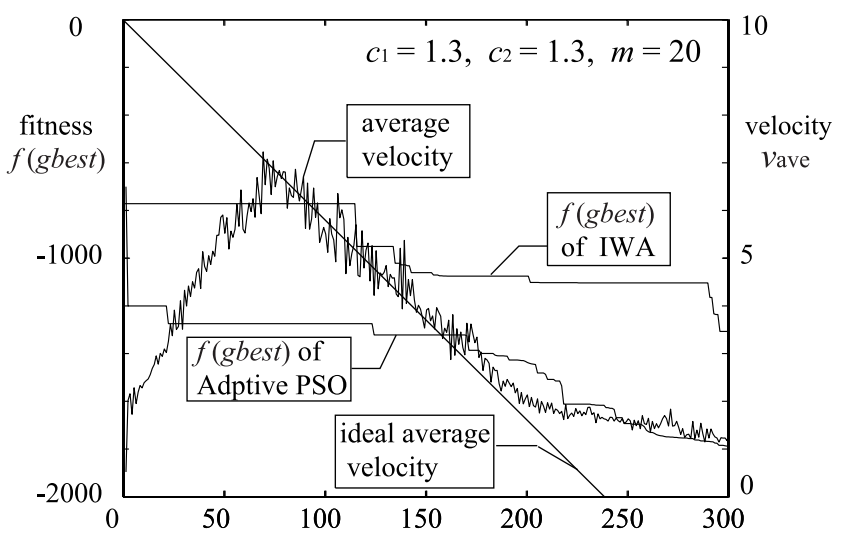

Fig. 2. Average velocity of swarm and fitness of the proposed adaptive PSO for $2^{n}$ minima $(n=30)$.

are used in this simulation. In comparison with IWA, the performance of the search of the proposed method for large-scale problems is considerably improved. The corresponding results for fitness of solution and $v_{\text {ave }}$ are shown in Fig. 2.

In Fig. 1, the proposed ideal average velocity reaches 0 before the end of searching, because it is intended to search intensively in the final stage of the search process. While the proposed method does not require that parameters be determined before the search begins, $\mathrm{V}_{\text {start }}$ in Fig. 1 must be determined. For the present problem, we selected $V_{\text {start }}=10$, because the search space for the $2^{n}$ minima is given by $-5 \leq x_{i} \leq 5$.

\section{Conclusions}

In this study we considered the qualitative and quantitative relationship between the behavior of the algorithm and the success or failure of the search. Based on the results of this analysis, we proposed an adaptive PSO that feeds back the information of average velocity of the swarm. The search ability of the proposed method was verified by simulation using a typical benchmark problem.

This work was supported by Japan Society for the Promotion of Science (JSPS) - a Grant-in-Aid for Scientific Research (C) (15560252).

(Manuscript received Oct. 28, 2004,

revised Feb. 22, 2005)

\section{References}

(1) J. Kennedy and R.C. Eberhart: "Swarm Interlligence", Morgan Kaufmann Publishers (2001) 